\title{
Algebraïscher Beweis des Satzes von der Anzahl der linearunabhängigen Integrale erster Gattung.
}

\author{
(Von E. B. Christoffer, in Strassburg.)
}

In meiner Abhandlung über die canonische Form der Integrale I. G. habe
ich mich auf einige bekannte Sätze bezogen und dabei angemerkt, dass die-
selben sich aus rein algebraïschen Betrachtungen schöpfen lassen. Der Wunsch,
dies näher zu erläutern und namentlich die algebraïschen Methoden nachzu-
weisen, deren ich mich bediene, veranlasst mich, im Folgenden meinen Beweis
von einem dieser Sätze mitzutheilen.
Der Gegenstand dieser Untersuchung gehört in die Grundlagen der Lehre
von den Abelschen Functionen, und betrifft einerseits die Anzahl der linear-
unabhängigen Integranden I. G. $w^{\prime}$, die zu einer gegebenen Gleichung
\[ F^{n}(s \mid z)=0 \]

gehören, andererseits die Kriterien für die Irreductibilität von $F$ resp. die Anzahl seiner irreductibeln Factoren.

Bevor ich zu dieser Untersuchung übergehe, muss ich befürworten, dass in dieser Materie mehrere Sätze von einander getrennt werden müssen. Ist die Gleichung (1) irreductibel und, indem ich mich zunächst der üblichen Bezeichnungen bediene, $r$ die Anzahl der Doppelpunkte $s|z=\gamma| \delta$, so lautet der erste Satz, dass die Anzahl der linearunabhängigen Functionen $w v^{\prime}$, welche zu dieser Gleichung gehören, stets gleich

$$
(m-1)(n-1)-r
$$

ist. Der zweite Satz ist von anderer Natur, und betrifft die zur Gleichung (1) gehörige Fläche $T$. Aus der Irreductibilität von (1) folgt, dass diese n-blättrige Fläche eine zusammenhängende ist, also ist die Anzahl der Doppellinien, in 
denen je zwei Blätter in einander übergehen, $\overline{>} n-1$. Bezeichnet man sie durch

$$
n-1+p
$$

so ist die Anzahl der einfachen Verzweigungspunkte $w=2(n-1+p)$, und da auch $w=2 m(n-1)-2 r$ ist, so folgt die bekannte Relation

$$
p=(m-1)(n-1)-r .
$$

Der erste Satz sagt also auch aus, dass die Anzahl der linearunabbängigen Functionen $w^{\prime}$ gleich ist der Anzahl $p$ der überzähligen, d. h. für den Zusammenhang von $T$ entbehrlichen Doppellinien dieser Fläche.

Der zweite Satz lehrt, dass (1) diese Fläche durch Querschnitte in eine einfachzusammenhängende verwandelt werden kann und dass dies (2) unter anderm durch $p$ Querschnittbündel $c, a, b$ (Riemanss, Theorie der Abelschen Functionen, § 19) geleistet wird.

Beide Sätze vereinigt geben den dritten Satz, dass die Anzahl der linearunabhängigen Functionen w' der Anzahl dieser Querschnittbündel gleich ist.

In dieser Form rührt der Satz von Riemans her; unabhängig vom DIRlchlet'schen Princip ist derselbe meines Wissens zuerst von Herrn PRYM bewiesen worden (Vergl. Borchardt's Journal 71, pag. 231-232); der Beweis folgt direct aus einem Riemans'schen Satze über die Periodicitätsmoduln der Integrale I. G. (Th. d. A. F., \& 21) wenn man berücksichtigt, dass der RIMans'sche Ausdruck von $w$ die Existenz von mindestens $p$ linearunabhängigen Functionen $w^{\prime}$ auf jeden Fall sicherstellt.

Der zweite von diesen Sätzen wird in der Lehre vom Zusammenhange der Fliiche $T$ mit selbstständigen Hülfsmitteln bewiesen; ein Gleiches kann man rom ersten Satze verlangen, und dann erst erhält dor so eben erwähnte Beweis des dritten Satzes seine wahre Bedeutung, da er in den Eigenschaften der Periodicitätsmoduln das Band nachweist, welches die bejden ersten, so sehr verschiedenartigen Lehrsätze direct miteinander verknüpft.

Von einem algebraïschen Beweise der ersten Satzes muss man hiernach fordern, dass er von der Querschnittstheorie unabhängig ist, aber ausserdem muss er die Irreductibilität der Gleichung (1), an die allein der Satz geknüpft ist, auch als den allein und unmittelbar entscheidenden Beweisgrund hervortreten lassen.

Beides leistet die folgende Untersuchung dadurch dass sie ron einer neuen Darstellung der Functionen $w^{\prime}$ ausgeht, und sie liefert ausserdem die Krite- 
rien für die Irreductibilität des Polynoms $F$ resp. die Anzahl seiner irreductibeln Factoren. Unmittelbar vorausgesetzt ist dabei der allgemeine Fall, den RIEMasn seinen Untersuchungen zu Grunde legt; der Schlussausdruck von $w^{\prime}$ ist indessen so beschaffen, dass man die Veränderungen überblicken kann, welche in ihm vorgehen, wenn sich beim Uebergange zu besondern Fällen höhere Singularitäten bilden.

Am Schlusse theile ich noch den doppelten Ausdruck einer Integralfunction $R$ mit, von welcher man sofort zu den Integralen III. und II. G. gelangt, sowie den Ausdruck einer algebraisschen wie $T$ verzweigten Function $S$ von $z$ mittelst des Integranden $R^{\prime}$.

I.

Sei in bekannter Bezeichnung

$$
F\left(s^{n} \mid z\right)=a s^{n}+a_{1} s^{n-1} \cdots+a_{n}=0
$$

eine Gleichung zwischen $s$ und $z$, und ihr Polynom $F$ entweder selbst irreductibel oder durch jeden irreductibeln Factor nur einmal theilbar; ausserdem sei $T$ die $n$-blättrige Fläche, durch deren Punkte man die Werthe von $z$ repräsentiren muss, damit $s$ denselben eindeutig zugeordnet werden kann, ohne an Linien (lignes d'arrêt nach $\mathrm{C}_{A \cup C H Y}$ ) unstetig zu sein. Iede Function $\sigma$ von $z$, welche diese Eigenschaft mit $s$ gemein hat, heisst verzweigt wie die Fläche $T$. Sind $s_{1} s_{2} \ldots s_{n}$ die Werthe von $s$ für das nämliche $z$, und bezichungs weise $\sigma_{1} \sigma_{2} \ldots \sigma_{n}$ die gleichzeitigen, den nämlichen Punkten von $T$ zugeordneten Werthe von $\sigma$, so kann man sich die Aufgabe stellen, im Ausdrucke

$$
\psi(t \mid z)=U+U_{1} t \cdots+U_{n-1} t^{n-1}
$$

die von $t$ unabhängigen Coefficienten $U, U_{i}, \ldots$ als Functionen von $z$ so zu bestimmen, dass für $i=1,2, \ldots n$

$$
\psi\left(s_{i} \mid z\right)=\sigma_{i}
$$

wird. Die Interpolationsformel von LaGRaNGE gibt sofort

wenn

$$
\psi(t \mid z)=\sum_{i=1}^{n} \frac{\sigma_{i}}{F^{\prime \prime}\left(s_{i} \mid z\right)} \cdot \frac{F(t \mid z)}{t-s_{i}},
$$

$$
F^{\prime}(t \mid z)=\frac{\partial F(t \mid z)}{\partial t}
$$


ist. Der vorstehende Ausdruck für $\psi$ ist unzulässig, wenn $F^{\prime}(s \mid z)$ identisch Null ist; aber dies ist durch die Bedingung ausgeschlossen, dass $F(t \mid z)$ durch keinen irreductibeln Factor zweimal aufgeht.

Zur Untersuchung von $\psi$ als Function von $z$ wird diese Variable ausser in $T$ auch noch in einer besondern Ebene $E$ repräsentirt. Beschreibt dann $z$ in dieser irgend einen in sich zurtickkehrenden Weg $l$, welcher durch keinen Verzweigungswerth $z$ von $s$ führt, so gibt dies in $T$ für den Punkt $z n$ allenthalben getrennte Wege $l_{1} l_{2} \ldots l_{n}$, deren Endpunkte demnach eine Permutation ihrer Anfangspunkte bilden. Die Endwerthe von $s_{1} s_{2} \ldots s_{n}$ und ebenso der zugeordneten Zweige $\sigma_{1} \sigma_{2} \ldots \sigma_{n}$ bilden die nämliche Permutation ihrer Anfangswerthe, folglich ist der Endwerth von $\psi$ dem Anfangswerthe gleich. Als Function von $\boldsymbol{z}$ hat also $\psi$ gar keine Verzweigungspunkte, also ist $\psi$ einwerthige Function von $z$ für jedes $t$, d. h. $U U_{1} \ldots$ sind einwerthige Functionen von $z$.

Iede wie $T$ verzweigte Function $\sigma$ von $z$ lässt sich also durch $s$ und $z$ in der Form

$$
\sigma=\psi(s \mid z)=U+U_{1} s \cdots+U_{n-1} s^{n-1}
$$

ausdrücken, wo die Coefficienten einwerthige Functionen von $z$ sind, und zwar liefert diese Gleichung zu jedem $Z$ weige von $s$ den ihm zugeordneten Zweig von $\sigma$.

Wird insbesondere $\sigma$ weder unendlich oft noch je zu unendlich hoher Ordnung unstetig, so überträgt sich dies auf $\psi(t \mid z)$, also ist dann $\psi(t \mid z)$ eine rationale Function von $z$.

II.

Wir stellen uns nun die Aufgabe, $\sigma=\psi(s \mid z)$ auf die allgemeinste Weise so zu bestimmen, dass

$$
w=\int \sigma d z=\int \psi(8 \mid z) d z
$$

ein Integral I. G., d. h. weder im Endlichen noch im Unendlichen jemals unstetig wird. Bei der Lösung dieser Aufgabe beschränken wir uns auf den Fall, wo 8 als Function von $z$ nur einfache und getrennte Singularitäten hat, und diese alle nur in Endlichen stattfinden d. h. wo (1) die Punkte in denen $s=\infty$ wird oder eine mehrfache Wurzel $s$ stattfindet, alle im Endlichen liegen und niemals zwei solcher Punkte für das nämliche $z$ stattfinden, ausserdem aber (2) als mehrfache Wurzeln nur Doppelwurzeln vorkommen. 
Es ist ein Fundamentalsatz, dass (1) dieser einfachste Fall für alle Grade $m, n$ der Gleichung (1) existirt und (2) wenn man ihn mit RIEMAN als den allgemeinen bezeichnet, jeder besondere Fall Grenzfall des allgemeinen ist, nämlich durch stetige Aenderung der Fläche $T$ und stetige Verlegung der Werthe von $s$ in ihr erreicht werden kann. Auch dieser wichtige Lehrsatz lässt sich mit rein algebraïschen Hülfsmitteln beweisen, wie es beim gegenwärtigen Stande der Lehre von den algebraïschen Functionen verlangt werden muss.

Im Uebrigen muss bemerkt werden, dass auch der directen Behandlung besonderer Fälle nach der hier auseinander zu setzenden Methode keine principiellen Hindernisse im Wege stehen.

Unter den vorstehenden Voraussetzungen nun darf $\sigma$ unstetig werden nur in den Verzweigungspunktèn, und zwar wie $1: \sqrt{z-\beta}$, wenn für $z=\beta$ ein solcher stattfindet; im Unendlichen muss auf jedem Blatte $\sigma_{1}^{\prime}=0^{2}$ werden, und umgekehrt sind diese Bedingungen auch ausreichend, damit das $\int \sigma d z$ nie unstetig wird also ein Integral I. G. ist.

Im gegenwärtigen Falle ist also

$$
\psi(t \mid z)=\sum_{i=1}^{n} \frac{\sigma_{i}}{F^{\prime}\left(s_{i} \mid z\right)} \cdot \frac{F(t \mid z)}{t-s_{i}}
$$

rationale Function von $z$, und wir haben zu untersuchen, wo und wie sie unstetig wird.

a) Nimmt $z$ einen solchen Werth an, dass $s_{i}=t$ wird, so bleibt der zweite Factor

$$
\frac{F(t \mid z)}{t-s i}
$$

stetig, da $t-s_{i}$ Wurzelfactor des Zählers ist. Dieser Factor wird also nur im Unendlichen unstetig, und zwar $=\infty^{m}$; aber dort wird auch $F^{\prime}\left(s_{i} \mid z\right)=\infty^{m}$ und $\sigma_{i}=0^{2} ;$ im Unendlichen wird also $\psi$ nicht unstetig, sondern unendlich klein zur zweiten Ordnung.

b) Die Unstetigkeitspunkte ron $\psi$ liegen also alle im Endlichen und sie entsprechen den Werthen von $z$, für welche in der Fläche $T$ der erste Factor

$$
\tau_{i}=\frac{\sigma_{i}}{F^{\prime}\left(s_{i} \mid z\right)}
$$

unendlich wird, also den Verzweigungspunkten von $T$ und den Doppelpunkten 
Der Vereinfachung wegen benutze ich nun für die Werthe, welche $s, z$ in den

$$
r=(m-1)(n-1)-p
$$

Punktepaaren, welche Doppelpunkte heissen, und in d'en

$$
w=2 m(n-1)-2 r=2(n-1+p)
$$

Verzweigungspunkten annehmen, die nämliche Bezeichnung

$$
s\left|z=\alpha_{i}\right| \beta_{i},
$$

so dass wir $w+r$ solcher Werthepaare zu berücksichtigen haben.

Ist alsdann $s\left|z=\alpha_{i}\right| \beta_{i}$ ein Verzweigungspunkt, so wird in ihm $\sigma=\infty$, $F^{\prime}=0$, aber $\sigma \sqrt{z-\beta_{i}}$ und $\frac{F^{\prime}}{\sqrt{z-\xi_{i}}}$ werden weder Null noch unendlich. Das nämliche gilt von $\left(z-\beta_{i}\right) \tau_{i}$, und zwar erlangt dieses Product im Verzweigungspunkte denselben Werth für beide Zweige von $\tau$, welche dort zusammenhängen. Bezeichnet man ihn durch $\frac{1}{2} A_{i}$, so folgt für $z=\beta_{i}$

$$
\lim \left(z-\beta_{i}\right) \psi(t \mid z)=A_{i} \frac{F\left(t \mid \beta_{i}\right)}{t-\alpha_{i}}
$$

für $z=\beta_{i}$ wird also $\psi$ unstetig und zwar

$$
\psi(t \mid z)=A_{i} \frac{F\left(t \mid \beta_{i}\right)}{t-\alpha_{i} z-\beta_{i}}+\text { funct. cont. }
$$

Findet für $z=\beta_{k}$ ein Doppelpunkt $s_{1}=s_{2}=\alpha_{k}$ statt, so bleiben $\sigma_{1}, \sigma_{2}$ stetig, aber $F^{\prime}\left(s_{1} \mid z\right), F^{\prime}\left(s_{2} \mid z\right)$ verschwinden wie $z-\beta_{k}$; also folgt für $z=\beta_{k}$

$$
\lim \left(z-\beta_{k}\right) \psi(t \mid z)=A_{k} \frac{F\left(t \mid \beta_{k}\right)}{t-\alpha_{k}},
$$

wo $A_{k}$ eine Constante ist. Für $z=\beta_{k}$ wird also $\psi$ eben falls unstetig und

$$
\psi(t \mid z)=A_{k} \frac{F\left(t \mid \beta_{k}\right)}{t-\alpha_{k} \cdot z-\xi_{k}}+\text { funct. cont. }
$$

Setzen wir daher den, bei Untersuchungen dieser Art stets wiederkehrenden Ausdruck

$$
\frac{F\left(t \mid \beta_{i}\right)}{t-\alpha_{i} \cdot z-\mu_{i}}=T_{i}(t \mid z)
$$

so folgt, dass $\psi(t \mid z)$ nur für die $w+r$ Werthe $\beta_{i}$ von $z$ unstetig wird, und für $z=\beta_{i}$

$$
\psi\left(t z=A_{i} T_{i}(t \mid z)+\right.\text { funct. cont. }
$$


ist. Der Ueberschuss von $\psi$ über die Summe aller Ausdrücke $A_{i} T_{i}$ wird ałso niemals unstetig, also ist er constant, und $=0$, da er im Unendlichen verschwindet. Es folgt

$$
\psi(t \mid z)=\sum_{i=1}^{10=r} A_{i} T_{i}(t \mid z)=\sum_{i=1}^{w 0=r} A_{i} \frac{F\left(t \mid \beta_{i}\right)}{t-\alpha_{i} \cdot z-\alpha_{i}} .
$$

Aber da $\psi$ im Unendlichen zur zweiten Ordnung verschwindet, so muss in seiner absteigenden Entwicklung das Glied mit $\frac{1}{z}$ fehlen, also

$$
\Sigma A_{i} \frac{F\left(t \mid \rho_{i}\right)}{t-\alpha_{i}}=0
$$

sein, und zwar für jedes $t$.

\section{III.}

Ietzt erhalten wir

$$
\sigma=\sum_{i} A_{i} T_{i}(s \mid z)=\sum_{i} A_{i} \frac{F\left(s \mid \beta_{i}\right)}{s-\alpha_{i} \cdot z-F_{i}}
$$

und haben zunächst zu untersuchen, in wie weit dieser Ausdruck den an $\sigma$ zustellenden Anforderungen genügt.

1) Im Unendlichen wird wegen $(A)$ auf jedem Blatte der Fläche $T$ : $n=0^{2}$, wie erforderlich ist.

2) Wir untersuchen demnach die Unstetigkeiten einer einzelnen Function

$$
T_{i}=\frac{F\left(s \mid \beta_{i}\right)}{s-\alpha_{i} \cdot z-\beta_{i}}
$$

nur noch für endliche Werthe von $z$.

a) Wenn beide Factoren des Nenners unendlich klein werden, so werden zwei Wurzelfactoren des Zählers $=s-\alpha_{i}$ bis auf Glieder von noch höherer Ordnung, also wird in diesen Falle

$$
T_{i}=\frac{s-\alpha_{i}}{z-\beta_{i}} G
$$

und an der Grenze $G$ weder Null, noch unendlich, noch $=\frac{0}{0}$.

Ist nun $\alpha_{i} \beta_{i}$ ein Verzweigungspunkt, so folgt, das $T_{i}$ in ihm unendlich wird wie $\frac{1}{\sqrt{z-\beta_{i}}}$, das $\int \sigma d z$ bleibt also dort stetig; das letztere gilt auch von den übrigen Functionen $T_{k}$. 
Entspricht dem Werthepaar $\alpha_{i} \beta_{i}$ ein Doppelpunkt, d. h. findet dasselbe in zwei getrennten Punkten der Fläche $T$ statt, so nimmt $G$, da es nicht $=\frac{0}{0}$ wird, in beiden Punkten den nämlichen Werth an, ebenso jede andere Function $T_{k}$; aber $\frac{s-\alpha_{i}}{z-\xi_{i}}$, welches in beiden Punkten ebenfalls stetig bleibt, erlangt in ihnen ungleiche Werthe, ebenso $T_{i}$. Letzteres und das $\int \sigma d z$ bleiben also stetig.

Hieraus ergibt sich der im Folgenden unentbehrliche Satz, dass die $w+r$ Functionen $T_{i}$ linearunabhängig sind. Sollte nämlich $T_{i}$ sich durch die übrigen Functionen $T_{k}$ linear und mit constanten Coefficienten ausdrücken lassen, so müsste, wenn $\alpha_{i} \beta_{i}$ ein Verzweigungspunkt ist, wenigstens eine der letztern dort unendlich werden, und wenn $\alpha_{i} \beta_{i}$ ein Doppelpunkt ist, wenigstens eine der übrigen Functionen $T_{k}$ in den entsprechenden Punkten von $T$ ungleiche Werthe annehmen, wovon weder das eine noch das andere der Fall ist.

b) Sodann ist der Fall zu untersuchen, wo nur ein Factor im Nenner von $T_{i}$ verschwindet. Wird $s=\alpha_{i}$ aber nicht $z=\beta_{i}$, so bleibt $T_{i}$ also $\sigma$ stetig, da $s-\alpha_{i}$ Wurzelfactor des Zählers von $T_{i}$ ist; wird $z=\beta_{i}$ aber $s$ nicht $=\alpha_{i}$, so wird $s$ eine andere Wurzel des Zählers, also abermals $T_{i}$ und $\sigma$ nicht unstetig.

c) Im Unendlichen sowie in allen denjenigen Punkten der Fläche $T$, in denen der Nenner einer der Functionen $T_{1}, T_{2}, \ldots$ verschwindet, hat also $\sigma$ alle verlangten Eigenschaften. Aber ausserhalb dieser Punkte wird noch jede Function $T_{i}$, und im Allgemeinen auch $\sigma$, unendlich so oft $s=\infty$ wird; soll also $\sigma$ auch in diesen Fällen stetig bleiben, so müssen seine $w+r$ Constanten $A_{1}, A_{2}, \ldots$ in geeigneter Weise beschränkt werden, und andere Beschränkungen derselben sind für unsere Aufgabe nicht erforderlich.

IV.

Sei

$$
Q(z)=\left(z-\beta_{1}\right)\left(z-\beta_{2}\right) \cdots\left(z-\beta_{w+r}\right),
$$

also $Q(z)$ der Ausdruck, welcher sich ergibt, wenn man die Discriminante $D$ der Gleichung (1) von dem Factor befreit, den sie mit ihrer Derivirten gemein hat. Dann ist $\psi(t \mid z) Q(z)$ eine ganze Function von $t$ und $z$, und von den 
Graden $n-1$ und $w+r-2$, da für $z==\infty \psi=0^{2}$ wird. Sei also

mithin

$$
\psi(t \mid z) \cdot Q(z)=G\left(\left(\left.^{n-1}\right|^{w+r-2} z\right)\right.
$$

$$
\sigma=\frac{G\left(\left.\left.s\right|^{n-1}\right|^{w+r-2}\right)}{Q(z)}
$$

wovon die Formeln der art. II. III. die Partialbruchzerfällungen sind.

Hier muss nun der Zähler $G$ ohne unnöthige Beschränkungen so bestimmt werden, dass er für $s=\infty$ niemals unstetig werden kann. Dies gelingt durch folgende Ueberlegungen. Schreibt man die Gleichung (1) in der Form

$$
a s^{\mu}+a_{1} s^{\mu-1} \cdots+a_{\mu}=-\frac{a_{\mu+1}}{s}-\frac{a_{\mu+2}}{s^{2}}-\cdots
$$

so folgt, für $\mu=0,1, \ldots n-1$, dass der Ausdruck zur Linken zur ersten Ordnung verschwindet, so oft $s=\infty$ wird. Wir setzen

$$
a t^{\mu}+a_{1} t^{\mu-1} \cdots+a_{\mu}=f_{\mu}(t \mid z)
$$

dann ist $f_{n}(s \mid z)$ identisch $=0$, die übrigen Functionen $f_{\mu}(s \mid z)$ werden $=0^{1}$ für $s=\infty$.

Eine ganze Function von $s$ und $z$, die in $s$ vom $\mu^{\text {ten }}$ Grade ist, wird im Allgemeinen $=\infty^{\mu}$ für $s=\infty$. Soll sie in jedem Falle, wo $s$ unendlich wird, entweder stetig bleiben oder doch nur zu einer kleinern als der $\mu^{\text {ten }}$ Ordnung unendlich werden, so muss der Factor von $s^{\mu}$ ohne Rest durch $a$ theilbar sein; ist er $=b \cdot a$, also auch $b$ ganze Function von $z$, so kann man das Glied höchster Ordnung

$$
b \cdot a s^{\mu}=b f_{\mu}(s \mid z) \text { - einer ganzen Function ron } s \text { und } z
$$

setzen, die in $s$ nur auf den Grad $\mu-1$ steigt; vereinigt man mit dieser die übrigen Glieder der in Rede stehenden ganzen Function, so erlangt sie die Ausdrucks form

$$
b f_{\mu}(s \mid z)+C_{1} s^{\mu-1}+\cdots+C_{\mu-1},
$$

wo $b, C_{1}, \ldots C_{\mu-1}$ ganze Functionen von $z$ sind.

Wendet man dies wiederholt auf die Function $G$ an, welche für $s=\infty$ niemals unstetig werden darf, so folgt, dass sie nothwendig die Form

$$
\begin{gathered}
G\left(\left.s^{n-1}\right|^{w+r-2} z\right. \\
\text { Annali di Matematica, tomo } \mathrm{X} \text {. }
\end{gathered}
$$


hat, wo $\Lambda_{0} \Lambda_{1} \ldots \Lambda_{n-1}$ ganze Functionen von $z$ sind, und zwar ist $\Lambda_{n-1}$ rom Grade $w+r-2$, alle übrigen sind nur vom Grade $w+r-m-2$.

Demnach haben wir nur noch die Bedingungen zu ermitteln, damit bei diesen Graden der Functionen $\Lambda$ identisch

$$
\psi(t \mid z)=\frac{1}{Q(z)}\left\{\sum_{\nu=0}^{n-2} \Lambda_{,}(z) f_{n-y-1}(t \mid z)+\Lambda_{n-1}(z)\right\}_{i=1}^{w+r} A_{i} \frac{F\left(t \mid \beta_{i}\right)}{t-\alpha_{i} \cdot z-\beta_{i}}
$$

wird. Dies gibt zunächst

$$
A_{i} \frac{F\left(t \mid \beta_{i}\right)}{t-\alpha_{i}}=\sum_{0}^{n-2} \frac{\Lambda_{\nu}\left(\beta_{i}\right)}{Q^{\prime}\left(\beta_{i}\right)} f_{n-y-1}\left(t \mid \beta_{i}\right)+\frac{\Lambda_{n-1}\left(\beta_{i}\right)}{Q^{\prime}\left(\beta_{i}\right)} .
$$

Nun ist, wenn $F(\alpha . \mid \Theta)=0$ ist,

$$
\frac{F(t \mid \beta)}{t-\gamma}=\sum_{\nu=0}^{n-1} \alpha^{\nu} f_{n-\nu-1}(t \mid \beta)
$$

berücksichtigt man, dass $f_{0}(\beta)=a(\beta)$ ist, so geht die vorige Formel über in

$$
A_{i} \sum_{0}^{n-2} \alpha_{i}^{\nu} f_{n-\nu-1}\left(t \mid \beta_{i}\right)+A_{i} \alpha_{i}^{n-1} a\left(\beta_{i}\right)=\sum_{0}^{n-2} \frac{\Lambda_{\nu}\left(\beta_{i}\right)}{Q^{\prime}\left(\beta_{i}\right)} f_{n-,-1}\left(t \mid \beta_{i}\right)+\frac{\Lambda_{n-1}\left(\beta_{i}\right)}{Q^{\prime}\left(\beta_{i}\right)} .
$$

Aber da $a\left(\beta_{i}\right)$ nicht $=0$ ist, so folgt aus der Gleichheit der Coefficienten von $t^{n-1}$ diejenige der Coefficienten von $f_{n-1}\left(t \mid \beta_{i}\right)=a\left(\beta_{i}\right) t^{n-1}+\cdots ;$ hebt man diese Glieder weg, so folgt ebenso die Gleichheit der Coefficienten von $f_{n-2}\left(t \mid \beta_{i}\right)$, u. s. w., so dass wir erhalten

$$
\left.\begin{array}{l}
\frac{\Lambda_{\nu}\left(\beta_{i}\right)}{Q^{\prime}\left(\beta_{i}\right)}=A_{i} \alpha_{i}^{\nu} \quad(\text { für } \nu=0,1, \ldots n-2) \\
\frac{\Lambda_{n-1}\left(\beta_{i}\right)}{Q^{\prime}\left(\beta_{i}\right)}=A_{i} \alpha_{i}^{n-1} a\left(\beta_{i}\right),
\end{array}\right\}
$$

beides für $i=1,2, \ldots w+r$. Daraus ergeben sich weiter die Partialbruchzerfällungen

$$
\begin{aligned}
& \frac{\Lambda_{\nu}(z)}{Q(z)}=\sum_{i} \frac{A_{i} \alpha_{i}^{\nu}}{z-\aleph_{i}} \quad(\text { für } \nu=0,1, \ldots n-2) \\
& \frac{\Lambda_{n-1}(z)}{Q(z)}=\sum_{i} \frac{A_{i} \alpha_{i}^{n-1} a\left(\beta_{i}\right)}{z-\xi_{i}} .
\end{aligned}
$$

Aus den Graden $w+r-m-2, w+r-2$ und $w+r$ von $\Lambda_{*}, \Lambda_{n-1}$ und $Q$ folgt, dass die absteigende Entwicklung in der ersten Formel mit $z^{-m-2}$, in der 
zweiten mit $z^{-2}$ beginnen muss; also folgt endlich

$$
\begin{aligned}
& \sum_{i} A_{i} \alpha_{i}^{\nu} \beta_{i}^{\mu}=0 \quad\left|\begin{array}{l}
\nu=0,1, \ldots \\
\mu=0,1, \ldots m
\end{array}\right| \\
& \sum_{i} A_{i} \alpha_{i}^{n-1} a\left(\beta_{i}\right)=0 .
\end{aligned}
$$

Sind umgekehrt diese Bedingungen erfüllt, so liefern die Gleichungen $(\gamma)$ jedes $\Lambda$ mit dem in ( $\alpha$.) vorgeschriebenen Grade, während $(\alpha)$ selbst auch ohne dies Folge von $(\beta)$ und dies Folge von $(\gamma)$ ist. Die vorstehenden Bedingungen $(B)$ $(C)$ sind also nothwendig und zugleich hinreichend, damit

$$
\psi(s \mid z)=\sum_{i} A_{i} \frac{F\left(s \mid \beta_{i}\right)}{s-\alpha_{i} \cdot z-\beta_{i}}
$$

für $s=\infty$ niemals unstetig wird.

$$
\text { V. }
$$

Jeder Integrand I. G. lässt sich also in die Form

$$
\sigma=\sum_{i=1}^{u+r} A_{i} \frac{F\left(s \mid \beta_{i}\right)}{s-\alpha_{i} \cdot z-\beta_{i}}=\sum_{i} A_{i} T_{i}(s \mid z)
$$

bringen, wo die $w+r$ Functionen $T_{i}$ linearunabhängig und ihre Coefficienten $A_{i}$ constant sind.

Aber es ist nicht jeder Ausdruck dieser Form ein Integrand I. G., sondern es sind hierzu die folgenden Bedingungen zwischen den Coefficienten $A_{i}$ erfor derlich und hinreichend:

$$
\begin{aligned}
& \sum_{i} A_{i} \frac{F\left(t \mid \beta_{i}\right)}{t-\alpha_{i}}=0 \quad \text { für jedes } t ; \\
& \sum_{i} A_{i} \alpha_{i}^{\nu} \rho_{i}^{\mu}=0 \\
& \sum_{i} A_{i} \alpha_{i}^{n-1} a\left(\beta_{i}\right)=0 .
\end{aligned}
$$

Dieselben sind jedoch nicht unabhängig voneinander. Die Gleichungen $(B)$ kann man durch die folgende ersetzen, dass für jede ganze Function $g\left(\begin{array}{c}n-2 \\ s \mid z\end{array}\right.$ 
sein muss

$$
\sum_{i} A_{i} g\left(\alpha_{i}^{n-2} \mid \beta_{i}\right)=0
$$

Nun ist $\frac{1}{n} F^{\prime}(s \mid z)=a(z) s^{n-1}+g\left(s^{n-2} \mid z\right)$, also folgt aus $(B)$ oder $(B 1)$

$$
\frac{1}{n} \sum_{i} A_{i} F^{\prime}\left(\alpha_{i} \mid \beta_{i}\right)=\sum_{i} A_{i} \alpha_{i}^{n-1} a\left(\beta_{i}\right)
$$

da $F^{\prime}\left(\alpha_{i} \mid \beta_{i}\right)=0$ ist, so ist $(C)$ eine Folge von $(B)$, also überzählig. Sodann ist

$$
f_{n-1}(s \mid z)=a s^{n-1}+g(\stackrel{n-2}{s \mid z})
$$

also können wir auch schreiben

$$
\frac{F(t \mid z)}{t-s}=f_{n-1}(s \mid z)+t f_{n-2}(s \mid z)+\cdots=a s^{n-1}+g\left(\begin{array}{c}
n-2 \\
s \mid z
\end{array}\right)
$$

mithin ist, als nothwendige Folge von $(B)$, auch

$$
\mathbf{\Sigma} A_{i} \frac{F\left(t \mid \beta_{i}\right)}{t-\gamma_{i}}=0
$$

und zwar für jedes $t$. In der That ist die Bedingung $(A)$, dass im Unendlichen $\psi(t \mid z)=0^{2}$ werden soll, auch durch die geforderten Grade der $\Lambda$ ausgedrückt.

Lassen wir die hiernach überzähligen Gleichungen $(A)$ und $(C)$ weg, so bleiben nur noch die Bedingungen $(B)$ übrig, und wir haben nun zu untersuchen $o b$ und unter welchen Bedingungen dieses System von überzähligen Gleichungen frei ist.

Angenommen, das System $(B)$ enthalte eine oder mehr als eine überzählige Gleichung. Dann kann man mit Benutzung von Multiplicatoren, die nicht alle $=0$ sind, aus $(B)$ alle Unbekannten $A_{i}$ eliminiren. Nimmt man diese Multiplicatoren zu Coefficienten der Function $g(s \mid z)$, so wird also in der Gleichung $(B 1)$ allgemein $g\left(\alpha_{i} \mid \beta_{i}\right)=0$, ohne dass alle Coefficienten von $g$ verschwinden. Es gibt also in diesem Falle eine ganze Function $g(\stackrel{n-2}{s \mid m})$, die in jedem Verzweigungs-und jedem Doppelpunkte verschwindet. Yon dieser Function $g$ erhalten wir also $w+2 r=2 m(n-1)$ Nullpunkte. Ich werde beweisen, dass $g$ von der Ordnung $2 m(n-1)$ ist; dann folgt, dass $g$ nur in jenen Punkten, und in keinem von ihnen zu einer höhern als der ersten Ordnung verschwindet. In der That wird in $n$ Fällen (für $z=\infty$ ) 
$g=\infty^{m}$, und in $m$ Fällen (für $s=\infty$ ) $g=\infty^{n-2}$, während sonst $g$ nicht mehr unstetig wird; also wird genau $n m+m(n-2)=2 m(n-1)$ mal $g=\infty$, wie so eben angegeben wurde.

Diese Null-und Unstetigkeitspunkte, nebst den nämlichen Ordnungszahlen, kommen auch der in jedem Falle wirklich existirenden Function $F^{\prime \prime}(s \mid z)$ zu; also ist $g: F^{\prime}=\gamma$ wie $T$ verzweigt, aber nie unstetig noch Null, also in jedem zusammenhängenden Theile von $T$ constant und in keinem $=0$. Vereinigt man jedesmal $\gamma$ mit $g$, so folgt:

Enthält das System $(B)$ eine oder mehr als eine überzählige Gleichung, so genügen diejenigen Wurzeln $s$ der Gleichung

$$
F(s \mid \stackrel{n}{z})=0
$$

welche dem nämlichen zusammenhängenden Theile der Fläche $T$ zugeordnet sind, auch einer Gleichung kleinern Grades

$$
g(\stackrel{n-2}{s \mid z})+F^{\prime}(s \mid z)=0,
$$

die ebenfalls in $z$ rational ist.

Hier trennen sich nun zwei Fälle voneinander.

A) Ist die Gleichung (1) irreductibel, so kann $s$ nicht auch noch der Gleichung (2) genügen; dann also ist es ein Widerspruch, anzunehmen, das System $(B)$ sei nicht frei von überzähligen Gleichungen. Die Anzahl der Gleichungen $(B)$ ist aber $(n-1)(m+1)=(m-1)(n-1)+2(n-1)=r+p+$ $+2(n-1)=r+w-p$, also um $p$ Einheiten kleiner als die Anzahl der Unbekannten $A_{i}$. Von diesen lässt sich also wenigstens eine Gruppe von $w+r-p$ Unbekannten $\left(^{*}\right)$ aus den Gleichungen $(B)$ ermitteln; sie drücken sich linear und homogen durch die $p$ übrigen aus, welche willkürlich bleiben, abgeschen vom Falle $p=0$, wo sämmtliche $A=0$ werden, und also ein Integral I. G. überhaupt nicht existirt. Sind $A_{1} \ldots A_{p}$ die Coefficienten, welche willkürlich bleiben, so erhält man für $k>p$ Auflösungsformeln

$$
A_{k}=\sum_{\rho=1}^{p} A_{\rho} \cdot \Delta_{k_{\rho}}
$$

(*) Eine genauere Bestimmung über diese Gruppe von Unbekannten ergibt sich am Schlusse des folgenden art. 
wo alle $\Delta$ Determinantenquotienten sind; setzt man alsdann den Gesammtfactor von $A_{\rho}$, nämlich

so wird

$$
T_{\rho}(s \mid z)+\sum_{l i p p} \Delta_{k_{p}} T_{k}(s \mid z)=w_{\rho}^{\prime}
$$

$$
\sigma=\psi(s \mid z)=A_{1} w_{1}^{\prime}+A_{2} w_{2}^{\prime} \cdots+A_{p} w_{p}^{\prime} .
$$

Hier sind demnach $w_{1}^{\prime} w_{2}^{\prime} \ldots w_{p}^{\prime}$ Integranden I. G., und sie sind linearunabhängig. Wären sie es nämlich nicht, so liesse sich wenigstens ein Anfangsglied $T_{\rho}$ durch andere Functionen $T_{i}$ linear und mit constanten Coefficienten ausdrücken, was unmöglich ist. Da der vorstehende Ausdruck alle Integranden I. G. enthält, so haben wir den Satz:

Ist die Gleichung

$$
F(\stackrel{n}{s} \mid \stackrel{m}{z)}=0
$$

irreductibel, so sind die $w+r-p$ Gleichungen

$$
\sum_{i=1}^{w+r} A_{i} \alpha_{i}^{\nu} \beta_{i}^{\mu}=0 \quad\left|\begin{array}{lc}
\nu=0,1, \ldots & n-2 \\
\mu=0,1, \ldots & m
\end{array}\right|
$$

voneinander unabhängig, und mit Benutzung derselben ist jeder, der Gleichung (1) zugeordnete Integrand I. G. in der Form

$$
\sigma=\sum_{i=1}^{w+r} A_{i} \frac{F\left(s \mid \beta_{i}\right)}{\left.s-\alpha_{i} \cdot z-\beta_{i}\right)}
$$

darstellbar; die Anzahl der linearunabhängigen Integranden I. G. ist in allen Fällen $=p$, insbesondere $=0$ für $p=0$.

$B)$ Enthält das System (B) überzählige Gleichungen, so kann die Gleichung (1) nicht irreductibel sein. Dieses Resultat ist unter der Voraussetzung hergeleitet, dass (art. I) das Polynom $F$ durch keinen irreductibeln Factor zweimal aufgehe, weil sonst $F^{\prime}(s \mid z)$ identisch Null ist: diese Bcdingung, an der wir festhalten, lässt sich auch so ausdrücken, dass die Discriminante $D$ von $F$ nicht identisch Null sein darf. Sei unter dieser Voraussetzung $k$ die Anzahl der irreductibeln, also ungleichen Factoren von $F$. Dann zerfällt die $n$-blättrige Fläche $T$ in $k$ zusammenhangende Flächen, den einzelnen Factoren von $\not{F}$ entsprechend, und jeder ist nach dem vorigen Satze eine Anzahl linearunabhängiger Integranden I. G. zugeordnet, gleich der An- 
zahl ihrer überzähligen Doppellinien. Die Anzahl aller der Gleichung (1) zugeordneten Integranden $\mathrm{I}$. $\mathrm{G}$. ist also gleich der Anzahl $p$ der überzähligen Doppellinien in dieser zerfallenden Fläche $T$. Für den Zusammenhang dieser Fläche sind unentbehrlich nur noch $(n-1)-(k-1)$ Doppellinien, also ist $(n-1)-(k-1)+p$ die Anzahl aller, und das Doppelte hiervon ist die Anzahl aller einfachen Verzweigungspunkte von $T$. Aber diese ist wieder $w=2 m(n-1)-2 r$, wenn $r$ die Anzahl aller Doppelpunkte ist, also folgt $(n-1)-(k-1)+p=m(n-1)-r$, d. i. $p=[(m-1)(n-1)-r]+k-1$ : die Anzahl der linearunabhängigen Integranden I. G. ist also um $k-1$ grösser wie in dem Falle, wo das System $(B)$ keine überzählige Gleichung enthält. Da diese Functionen unter Voraussetzung der Gleichungen $(B)$ alle im obigen Ausdrucke von $\sigma$ enthalten sind, so folgt, dass das System $(B)$ genau $k-1$ überzählige Gleichungen enthält. Dies lässt sich umkehren:

Enthält das System $(B) k-1$ überzählige Gleichungen, und ist die Discriminante $D$ von $F$ nicht identisch Null, so zerfällt $F$ in $k$ ungleiche irreductible Factoren.

Denn wäre $F$ durch denselben irreductibeln Factor zweimal theilbar, so wäre $D$ identisch Null; wäre $F$ das Product aus $l$ ungleichen, irreductibeln Factoren, und $l$ nicht $=k$, so enthielte das System $(B) l-1$ und nicht $k-1$ überzählige Gleichungen.

VI.

Nunmehr ist

$$
\varphi(t \mid z)=\sum_{i=1}^{n} \sigma_{i} \frac{F(t \mid z)}{t-s_{i}}
$$

ganze Function von $t$ und rationale Function von $z$. Für $s_{i}=t$ bleibt sic stetig, ebenso wenn $\sigma=\infty$ wird. Denn wenn dies für $z=\beta$ stattfindet, so wird $(z-\beta) \sigma=0$, also auch $(z-\beta) \varphi(t \mid z)=0$, mithin $\varphi$ nicht unstetig. Da hiernach die rationale Function $\varphi$ für kein endliches $z$ unstetig wird, so ist sie ganze Function von $z$. Für $z=\infty$ findet sich $\varphi=\infty^{m-2}$, also ist $\varphi$ in $z$ vom Grade $m-2$. Ist, nach $t$ geordnet, $\varphi=C t^{n-1}+C_{1} t^{n-2}+\cdots$, so wird der leitende Coefficient

$$
C=a \Sigma \sigma_{i}
$$


derselbe ist gleich Null, denn die $\mathbf{\Sigma} \sigma_{i}$ ist ebenfalls rational, im Endlichen nie unstetig und im Unendlichen Null, also identisch Null. Folglich ist $\varphi$ in $t$ nur rom Grade $n-2$, und wir erhalten

$$
\sum_{i=1}^{n} \sigma_{i} \frac{F(t \mid z)}{t-s_{i}}=\varphi\left(\left.t^{n-2}\right|^{m-2} z\right)
$$

Findet für $z=\delta$ ein Doppelpunkt $s_{1}=s_{2}=\gamma$ statt, so hat $F(t \mid d)$ den Factor $t-y$ zweimal, in zwei Gliedern hebt er sich aber einmal weg, also hat $\varphi(t \mid \delta)$ ihn einmal. Für $\sigma$ ergibt sich der bekannte Ausdruck RiEnanss

$$
\sigma F^{\prime}(s \mid z)=\varphi\left(\left.\stackrel{n-2}{s}\right|^{m-2} z\right)
$$

mit dem Zusatze, dass $\varphi(s \mid z)$ in den $r$ Doppelpunkten verschwindet; es ist ebenfalls bekannt, dass diese Eigenschaft auch ausreicht, damit $\sigma$ Integrand I. G. wird. Zwischen den $(m-1)(n-1)$ Coefficienten von $\varphi$ ergeben sich also in Rremanx'scher Bezeichnung die $r$ Bedingungsgleichungen

$$
\varphi\left(\gamma_{\rho} \mid \delta_{\rho}\right)=0 \quad(\rho=1,2, \ldots r) ;
$$

ist $l$ die Anzahl der überzähligen Gleichungen dieses Systems, so ergeben sich $[(m-1)(n-1)-r]+l$ Functionen $\sigma$; dieselben sind linearunabhängig, da $\varphi$ nicht durch alle irreductibeln Factoren von $F$ theilbar sein kann.

Ist die Gleichung (1) irreductibel, so ist $l=0$ :

Ist die Gleichung (1) irreductibel, so ist die Anzahl $r$ und die Lage der Doppelpunkte $\gamma \delta$ eine solche, dass unter den $r$ Gleichungen $\left(B^{\prime}\right)$ sich keine überzählige findet.

Dazu kommt wie am Schlusse des vorigen art. der Satz:

Enthält das System $\left(B^{\prime}\right)$ vermöge der Anzahl $r$ und der Lage der Doppelpunkte überzählige Gleichungen, und ist die Anzahl derselben $=k-1$, so zerfällt $F$ in $k$ ungleiche irreductible Factoren, vorausgesetzt dass seine Discriminante nicht identisch $\mathrm{Null}$ ist.

Die gegenwärtige Untersuchung zeigt, dass in allen diesen Fällen ein Integrand I. G. $w^{\prime}$ in den beiden Punkten von $T$, die einem Doppelpunkte entsprechen, ungleiche Werthe annimmt; stellt man also $w^{\prime}$ durch Functionen $T$ dar, so muss sein Ausdruck alle Functionen $T_{k}$ enthalten, welche einem Doppelpunkte $\alpha_{k} \beta_{k}$ zugeordnet sind. 
Die entsprechenden Coefficienten $A_{k}$ gehören daher auf alle Fälle zu derjenigen Gruppe von Unbekannten, nach denen das System $(B)$ aufgelöst werden kann,

denn wenn $A_{k}$ willkürlich bleibt, kommt $T_{k}$ nur in einer Function $w_{k}^{\prime}$ vor.

VII.

Auf diesen Sätzen beruht die Möglichkeit der Integrale III. und II. G. für jedes $p$, auch für $p=0$. Ich finde in dieser Bezichung folgende Resultate, deren Beweis ich nach dem Vorangehenden wohl übergehen darf. Sei $\varepsilon$ ein von $s \mid z$ unabhängig veränderlicher Punkt der Fläche $T$ und in ihm $z=\zeta$, $s=\sigma$, ferner, wenn wieder $\alpha_{i} \beta_{i}$ einen Verzweigungs oder Doppelpunkt bedeutet

$$
\mathfrak{A}(\varepsilon)=\sum_{i=1}^{w+r} A_{i}(\varepsilon) \frac{F\left(s \mid \beta_{i}\right)}{s-\alpha_{i} \cdot z-\psi_{i}}-\frac{1}{F^{\prime}(\sigma \mid \zeta)} \frac{F(s \mid \zeta)}{s-\sigma \cdot z-\zeta},
$$

während die $w+r$ Constanten $A_{i}(\varepsilon)$ an die $w+r-p$ Gleichungen

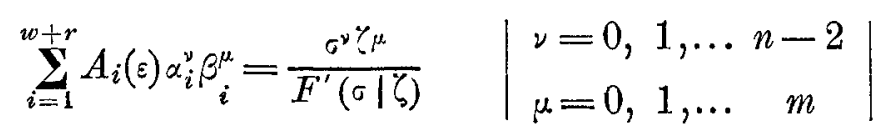

gebund en sind. Wir sctzen voraus, dass die Gleichung (1) irreductibel ist, dann folgt aus art. $V$., dass diese Gleichungen niemals einander widersprechen können, und $w+r-p$ Unbekannte $A_{i}$ durch die $p$ übrigen und die unabhängigen Glieder ohne Widerspruch bestimmen. Insbesondere ist also $\mathfrak{A}(\varepsilon)$ bestimmt bis auf einen additiven Integranden I. G. Durch die vorstehenden Bedingungen ist also auch die Integralfunction

$$
R(\varepsilon)=\int \mathfrak{H}(\varepsilon) d z
$$

widerspruchsfrei bestimmt bis auf ein additives Integral I. G. Dieselbe hat die folgenden Eigenschaften:

1. Im Punkte $\varepsilon$ ist

$$
R(\varepsilon)=-\log (z-\zeta)+\text { funct. cont. mon. }
$$

2. In jedem der $n$ unendlich fernen Punkte $\infty_{\text {y }}$ von $T$ ist

$$
R(\varepsilon)=\frac{1}{n} \log \frac{1}{z}+\text { funct. cont. mon. }
$$


3. Dies sind die einzigen Punkte, in denen diese Function unendlich wird.

Um sie eindeutig zu machen, verwandle man daher die Fläche $T$ mittelst der bekannten Querschnittbündel $c, a, b$ in eine einfach zusammenhängende Fläche $T^{\prime}$, und ziche durch diese aus dem nämlichen Punkte, von dem alle Schnitte $c$ nach den Schnittpaaren $a, b$ ausgehen, nacheinander noch einen Schnitt $l$ bis $\varepsilon$ und Schnitte $l_{1} l_{2} \ldots l_{n}$ nach den unendlich fernen Punkten $\infty_{1} \infty_{2} \ldots \infty_{n}$. Wird der Integrationsweg auf diese, ebenfalls noch einfach zusammenhängende Fläche $T_{1}$ beschränkt, so wird $R$ eindentig und im Innern von $T_{1}$ nie unstetig; die Periodicitätsmoduln dieser Function sind alle constant. Bezeichnet man die Seiten der Querschnitte $a, b$ in üblicher Weise und die Seiten der Schnitte $l$ so, dass man durch einen positiven Umlauf um den Endpunkt von der negativen auf die positive Seite des Schnittes gelangt, so ist

$$
\text { 4. } \begin{array}{ll}
\text { an } l & \vec{R}-\vec{R}=-2 \pi i \\
\text { an } l_{1} l_{2} \ldots l_{n} & \stackrel{+}{R}-\bar{R}=\frac{2 \pi i}{\imath} .
\end{array}
$$

Ist sodann $u_{\mu}$ das $\mu^{\text {te }}$ Normalintegral I. G. und

5. an $a_{\lambda}$

$$
\begin{array}{lll}
\text { an } a_{\lambda} & \stackrel{+}{R}-\bar{R}=A_{\lambda}(\varepsilon) & + \\
& +u_{\mu}-\bar{u}_{\mu}=\left(\begin{array}{l}
\lambda \\
\mu
\end{array}\right) \pi i \\
\text { an } b_{\lambda} & +\vec{R}-\bar{R}=B_{j}(\varepsilon) & +u_{\mu}-\bar{u}_{\mu}=a_{\lambda_{\mu}},
\end{array}
$$

während an $c_{\text {i }}$ beide Functionen stetig sind, so folgt aus der Untersuchung der Function $\int R d u_{\mu}$ in bekannter Weise

$$
\text { 6. } \quad B_{\mu}(\varepsilon)=\frac{1}{\pi \imath} \sum_{\mu=1}^{p} A_{\lambda}(\varepsilon) \alpha_{j \mu}-2 u_{\mu}(\varepsilon)+\frac{2}{n} \sum_{\nu=1}^{n} u_{\mu}\left(\infty_{\nu}\right) .
$$

Hier bedeuten $u_{\mu}(\varepsilon), u_{\mu}\left(\infty_{\nu}\right)$ die Werthe von $u_{\mu}$ in den Punkten $\varepsilon, \infty$, von $T^{\prime}$; unter dem Symbol $\left(\begin{array}{l}\lambda \\ \mu\end{array}\right)$ verstehe ich die Einheit, wenn $\lambda=\mu$ ist, in allen ïbrigen Fällen die Null $\left(^{*}\right)$.

(*) Ein ähnliches Symbol fur die Zwecke der Determinantentheorie findet sich in der Literatur zuerst in einer Abhandlung des Herrn Krosecker (Monatsber. d. Berliner Ac. vom 15 Oct. 1866, pag. 601); ich selbst bin vor langer Zeit zum obigen Symbol durch Untersuchungen genöthigt worden, in denen Derivirten wie $\frac{\partial x_{\nu}}{\partial x_{\mu}}=\left(\begin{array}{l}\lambda \\ p\end{array}\right)$ nicht zu vermeiden sind. während $x_{1} x_{2} \ldots x_{n}$ unabhängige Variabeln bedeuten. 
Die Differenz zweier Functionen $R$ ist demnach ein Integral III. G.,

$$
R\left(\varepsilon_{1}\right)-R\left(\varepsilon_{2}\right)=\varpi\left(\varepsilon_{1} \mid \varepsilon_{2}\right)
$$

und

$$
t(\varepsilon)=\frac{\pi R(\varepsilon)}{a \zeta}
$$

ein Integral II. G. (Riemanss: Th. d. A. F., art. 4.)

Die nämliche Function $R$ lässt sich auch in der folgenden Form dars:ellen

$$
R(\varepsilon)=\int\left\{\Phi\left(\begin{array}{c}
n-2 m-2 \\
s \mid z)
\end{array}-\frac{F(s \mid \zeta)}{s-\sigma \cdot z-\zeta}-\frac{1}{n} \frac{F^{\prime}(s \mid z)-F^{\prime}(s \mid \zeta)}{z-\zeta}\right\} \frac{\lambda z}{F^{\prime}(s \mid z)},\right.
$$

wofern die $(m-1)(n-1)=r+p$ Constanten von $\Phi$ so bestimmt werden, dass der Ausdruck zwischen den gewundenen Klammern in den $r$ Doppelpunkten verschwindet. Nach art. VI. sind diese $r$ Bedingungsgleichungen widerspruchsfrei und voneinander unabhängig; also ist auch in dieser Form $R$ völlig bestimmt bis auf ein additives Integral I. G.

Um von den Anwendungen dieser Function $R$ nur ein Beispiel anzudeuten, sei $S$ eine algebraïsche wie $T$ verzweigte Function von $z$, die nur im Endlichen und zwar in den $q$ Punkten $\varepsilon_{1} \varepsilon_{2} \ldots \varepsilon_{q}$ unstetig wird. Der Einfachheitwegen beschränken wir uns auf den Fall, wo $S$ nur zur 1. Ordnung unendlich wird, und sei

$$
\text { in } \varepsilon_{k}: \quad S=\frac{E_{k}}{z-\zeta_{k}}+\text { funct. cont. } \quad(k=1,2, \ldots q),
$$

wenn $\zeta_{k}$ den Werth von $z$ in $\varepsilon_{k}$ und $E_{k}$ eine Constante bedeutet. Dann ergibt sich die wirkliche Darstellung von $S$ als rationale Function von $s$ und $z$ wie folgt. Ist $w$ irgend ein Integral I. G. und seine Derivirte $\frac{d w}{d z}$ in $\varepsilon_{k}$ gleich $w^{\prime}\left(\varepsilon_{k}\right)$, so ist bekanntlich

$$
\sum_{k=1}^{q} E_{k} w^{\prime}\left(\varepsilon_{k}\right)=0
$$

Nun bilde man $I=\int S d w, \Delta=\sum_{k} E_{k} w^{\prime}\left(\varepsilon_{k}\right) R\left(\varepsilon_{k}\right)$, beides Integrale algebraïscher wie $T$ verzweigter Functionen von $z$. Dann bleibt $I$, und wegen $(\alpha)$ auch $\Delta$ im Unendlichen stetig, im Endlichen werden sie beide unendlich nur in den Punkten $\varepsilon$, aber wie man sofort sicht so, dass ihre Summe $I+\Delta$ auch dort stetig bleibt. Letztere ist also ein allenthalben endliches Integral, also ein In- 
tegral I. G. $c_{1} w_{1}+c_{2} w_{2} \cdots+c_{p} w_{p}+$ const.; daraus folgt

$$
S w^{\prime}=c_{1} w_{1}^{\prime}+c_{2} w_{2}^{\prime} \cdots+c_{p} w_{p}^{\prime}-\sum_{k=1}^{q} E_{k} w^{\prime}\left(\varepsilon_{k}\right) \frac{d R\left(\varepsilon_{k}\right)}{d z} .
$$

Hier sind noch die $p$ Constanten $c_{1} c_{2} \ldots c_{p}$ zu bestimmen, was sofort zu meiner Unterscheidung von Functionen $S$ der I. und der II. G. führt.

Es ist unnöthig: die Modificationen zu erläutern, welche sich im Ausdrucke von $S$ ergeben, wenn $S$ in einem Punkte $\varepsilon$ zu höherer Ordnung unendlich wird, oder im Ausdrucke und den Unstetigkeiten von $R(\varepsilon)$, wenn $\varepsilon$ in einen unendlich fernen Punkt $\infty$, rückt; der erste von den obigen Ausdrücken von $R$ liefert die Entscheidung der letztern Frage ohne Weiteres.

Strassburg, 18 Februar 1880. 